\title{
Incontinence anale
}

\section{Conclusion}

\author{
A. Senéjoux · J.D. Zeitoun \\ (C) Springer-Verlag France 2012
}

Ce dossier thématique souligne que l'incontinence anale est une affection fréquente, souvent inavouée. Les étiologies de cette affection sont multiples, souvent associées entre elles. La continence fait en effet intervenir plusieurs éléments : appareil sphinctérien, système nerveux, réservoir rectal, qualité des selles... Les troubles du transit sont très souvent associés à l'incontinence, et leur correction constitue la première approche thérapeutique de l'affection, efficace chez plus d'un malade sur deux. En cas d'échec, les possibilités thérapeutiques sont multiples, à adapter au mécanisme physiopathologique de l'incontinence. Certaines sont relativement « douces » pour les malades (rééducation périnéale,

TENS...) d'autres plus lourdes, potentiellement grevées d'une morbidité et au mieux réalisées en centre expert.

Ce dossier thématique aborde les différents traitements disponibles et aussi les terrains prédisposés sur lesquels peut survenir l'incontinence (post partum, malade âgé, patient neurologique), le traitement choisi étant bien entendu à adapter au cas par cas.

Le résultat du travail de la SNFCP concernant la prise en charge de l'Incontinence anale devrait bientôt être présenté et publié, gageons qu'il apportera encore des éclaircissements supplémentaires sur les modalités de prise en charge optimales de cette affection complexe.

\footnotetext{
A. Senéjoux $(\square)$

Centre Hospitalier Privé Rennes Saint Grégoire, 6, boulevard de la Boutière, F-35760 Saint Grégoire, France

e-mail : agnes.senejoux@gmail.com

J.D. Zeitoun $(\bowtie)$

Centre hospitalier Diaconesses-Croix Saint-Simon, service de proctologie médico-interventionnelle, 18, rue du Sergent Bauchat, F-75012 Paris, France

e-mail : jdzeitoun@yahoo.fr
} 